\title{
La pseudogestation chez la chèvre
}

\author{
R Duquesnel 1, D Parisot, G Pirot 2, JP Mialot, \\ L Saboureau, P Étienne 1, J Delaval 4, JM Guéraud, \\ E Prengere 5, G de Montigny 6, P Guerrault, G Perrin 7, \\ $P$ Humblot $8, Y$ de Fontaubert, $P$ Chemineau 9
}

\author{
1 URGDS du Centre, BP 501, 36018 Châteauroux cedex; \\ 2 GDS du Cher, 216 rue Louis-Mallet, 18000 Bourges; \\ ${ }^{3}$ ENV d'Alfort, productions animales, 7 avenue du Gal-de-Gaulle, 94704 Maisons-Alfort cedex; \\ ${ }^{4}$ Laboratoire vétérinaire départemental de I'Indre, BP 617, 36020 Châteauroux cedex; \\ ${ }^{5}$ Laboratoire vétérinaire départemental du Cher, 216 rue Louis-Mallet, 18000 Bourges; \\ 6 Institut de l'Élevage, 149 rue de Bercy, 75595 Paris cedex 12; \\ 7 Station régionale de pathologie caprine, BP 3081, 79012 Niort cedex; \\ 8 UNCEIA, 13 rue Jouët, 94700 Maisons-Alfort; \\ 9 INRA, physiologie de la reproduction, 37380 Nouzilly, France
}

(Reçu le 21 septembre 1991; accepté le 15 novembre 1991)

\begin{abstract}
Résumé - Une enquête échographique réalisée en région Centre en 1989 et 1990 sur un échantillon de plus de 10000 chèvres permet d'évaluer l'incidence globale de l'affection entre 2 et $3 \%$ des animaux répartis dans plus de $55 \%$ des 139 élevages enquêtés. Cependant l'incidence dépasse $5 \%$ des femelles échographiées après mise à la reproduction dans plus de $10 \%$ des élevages. L'affection s'observe à tout âge et plus rarement sur des chevrettes âgées de plus de 6 mois. On observe significativement plus de cas de pseudogestation chez les chèvres désaisonnées et chez les chèvres soumises à la synchronisation de l'œstrus. L'autopsie de 8 chèvres et 2 chevrettes diagnostiquées pseudogestantes par échographie a permis d'observer un utérus distendu contenant 1-7,2 I de liquide translucide stérile. Les ovaires présentent toujours un ou plusieurs corps jaunes mais également des follicules de De Graaf. La progestéronémie est élevée.
\end{abstract}

\section{chèvre / pseudogestation / progestérone / saison / gestation}

Summary - Pseudopregnancy in the goat. A large echographic investigation carried out in the centre of France on a sample of over 10000 she-goats allowed the incidence of the disorder to be evaluated at between 2 and $3 \%$ of the females spread over $>55 \%$ of the flocks. However the incidence was $>5 \%$ in $11 \%$ of the flocks. The disorder was observed at all ages and more rarely in female kids > 6 months old which had never mated with a male. Significantly more cases of pseudopregnancy were observed in out-of-season goats after kidding in autumn and in goats submitted to hormonal treatments for synchronization of oestrus. Autopsy of 6 she-goats and 2 female kids diagnosed as pseudopregnant by echography showed a distended uterus containing 1 to 7.21 of transparent sterile liquid. Ovaries still bore one or more corpus luteum but also Graafian follicles. Progesteronemia was elevated. 


\section{INTRODUCTION}

Avec environ $47010^{6}$ I produits en 1989 (collecte + fabrication de fromage à la ferme) pour un effectif de 900000 chèvres, la France est le premier pays producteur de lait de chèvre de la CEE à égalité de volume avec la Grèce et l'Espagne. En 20 ans, les élevages caprins spécialisés de plus de 50 chèvres se sont développés. Alors qu'en 1970, ils ne représentent que $1 \%$ des élevages et $12 \%$ de l'effectif de chèvres, en 1988 ils représentent $9 \%$ des élevages et détiennent $52 \%$ du troupeau caprin national. Cette augmentation de la taille des troupeaux s'est accompagnée d'une modification et d'une rationalisation des techniques de conduite des élevages.

La synchronisation des chaleurs et l'avancement de la période des saillies sont fréquemment réalisés sur des lots de chèvres par le recours à la pose d'éponge vaginale du mois de mai au mois de juillet, alors que naturellement les chèvres présentent un œstrus du mois d'octobre au mois de février (Chemineau, 1989).

Le diagnostic de gestation par échographie s'est développé ces dernières années dans l'élevage caprin (Tainturier et al, 1983; de Montigny, 1985; Cartier, 1986; Jardon et Pelzer, 1988; Mialot et al, 1991a). Cette technique a permis d'établir fréquemment le diagnostic de la pseudogestation, affection qui n'était décrite qu'occasionnellement auparavant (Lyngset, 1968; Faudell, 1979; Smith, 1980; Mews, 1981; Pieterse et Taverne, 1986; Malher et Younes, 1987; Taverne et al, 1988; Freeman, 1989; Saboureau, 1990; Etienne, 1991; Mialot et al, 1991a,b). Plusieurs synonymes sont utilisés : hydromètre, mucomètre, cloud burst (trombe d'eau).

Dans les élevages, cette affection existe depuis longtemps. Les éleveurs parlent de chèvres qui "font de l'eau" ou de chèvres qui "coulent" et d'un certain nombre de chèvres qui "font de l'eau ou du sang". Cette description rend compte de la vidange spontanée de l'utérus.

\section{ÉTUDE CLINIQUE}

\section{Définition}

La pseudogestation se caractérise par:

- une accumulation de liquide stérile en grande quantité dans l'utérus, associée à un corps jaune persistant sur les ovaires;

- une augmentation du volume de l'abdomen et une absence de manifestation de l'œstrus pendant plusieurs semaines voire plusieurs mois;

- l'absence d'embryon, de vésicule embryonnaire et de placenta dans l'utérus;

- dans certains cas, un écoulement spontané ou provoqué du liquide utérin avec une diminution du volume de l'abdomen.

\section{Epidémiologie descriptive}

\section{L'âge des animaux}

La pseudogestation s'observe la plupart du temps dans le mois qui suit la mise à la reproduction car c'est à ce moment que les échographies sont pratiquées en routine dans les élevages en vue du diagnostic de gestation. Néanmoins, son apparition concerne également des chèvres dans les mois qui suivent la mise-bas avant mise à la reproduction. Ainsi, dans un élevage Saanen, 12 des 96 chèvres ayant mis-bas à contre saison en novembre $1989(13 \%)$ et 9 des 76 chèvres ayant misbas en novembre $1990(12 \%)$ présentaient un état de pseudogestation détectable par 
échographie de mars à mai, 4-6 mois après mise-bas et avant toute mise à la reproduction.

D'autre part, des chevrettes n'ayant jamais mis-bas peuvent également présenter cette affection après leur $1^{\text {re }}$ mise à la reproduction. Ainsi, dans un élevage alpin traditionnel avançant la mise à la reproduction du troupeau en posant des éponges en mai et juin sur une partie des chèvres adultes, 2 des 16 chevrettes (13\%) présentaient une pseudogestation détectable en décembre 90 à l'âge de 1 an.

Des cas similaires ont été observés dans un élevage sur un lot de chevrettes d'un an non soumis à la saillie par un bouc.

Enfin, de très mauvais résultats de fécondité sur des lots d'animaux inséminés après pose d'éponge en mai, juin, juillet peuvent s'expliquer par un taux important de chèvres pseudogestantes au moment de la mise à la reproduction. Du fait de l'administration de prostaglandines dans le cadre du protocole de l'insémination, les échographies réalisées après insémination ne détectent plus cette affection. Ainsi, dans un élevage pratiquant la monte en main et l'insémination à contre saison, les taux de réussite en IA ont été de $28 \%$ de mise-bas pour 28 IA en 1988 et $38 \%$ de mise-bas pour 21 IA en 1989. Les pseudogestations ont été mises en évidence uniquement sur un lot d'animaux soumis à la monte en main à la même période après synchronisation de l'œstrus par pose d'éponge sans utilisation de prostaglandine. Dans ce lot, $31 \%$ des 42 chèvres présentaient un état de pseudogestation 1 mois et demi après saillie.

\section{Évolution dans le temps au sein d'un même élevage}

\section{Au cours de l'année}

Cette affection peut débuter à 2 périodes distinctes. Nous avons observé que la pseudogestation s'installe sur certaines chèvres dans les mois qui suivent la misebas quand celle-ci a lieu précocement en novembre ou décembre. Par la suite, la pseudogestation peut s'interrompre spontanément sur un certain nombre de ces animaux en février, en mars ou avril. Mises à la reproduction, certaines chèvres sont fécondées début août. Chez d'autres chèvres, en l'absence de traitement, la pseudogestation se poursuit jusqu'en juillet-août. Certaines chèvres ne se vident que partiellement. Chez d'autres, l'affection récidive l'automne suivant.

Certains éleveurs prolongent ainsi des chèvres en lactation 2 ou 3 années de suite sans mise à la reproduction, utilisant uniquement un traitement de prostaglandine pour les «vider». Économiquement une chèvre pseudogestante n'est donc pas inintéressante si elle reste en lactation. Aussi des échographies sont également réalisées dans certains élevages pour ne pas tarir les chèvres pseudogestantes par ignorance.

D'autre part nous avons observé que la pseudogestation peut débuter sur des animaux ayant mis-bas en saison normale (février, mars, avril) après leur mise à la reproduction, lorsque celle-ci est artificiellement avancée au mois de juillet par pose d'éponge, par "effet bouc» ou par «effet femelle». Nous avons alors observé cette affection par une échographie réalisée au mois de septembre.

\section{Évolution en fonction des années}

Dans un même élevage, le nombre de chèvres atteintes peut être extrêmement variable d'une année à l'autre. Ainsi, dans un même élevage l'incidence des chèvres pseudogestantes est passée de $26 \%$ en septembre 1989 (73 chèvres échographiées après mise à la reproduction) à $2 \%$ en septembre 1990. Par ailleurs, dans un élevage Saanen, l'incidence de l'affection un mois après mise à la reproduction est 
passée de $0 \%$ du lot des 36 chèvres inséminées en juillet 1989 à 3 chèvres sur 25 (12\%) du lot inséminé en juillet 1990 suivant le même protocole. Aucun changement dans l'alimentation ou dans la conduite de l'élevage n'est intervenu entre ces 2 années.

\section{Diagnostics}

\section{Diagnostic clinique}

Durant la phase d'installation puis d'état de pseudogestation aucune manifestation clinique ne la différencie d'un état de gestation vraie. La chèvre ne présente pas de manifestation cestrale. On peut observer parfois une augmentation du volume de l'abdomen. Certains éleveurs relatent même en fin d'évolution une diminution de la lactation. $\mathrm{Si}$ cette affection survient après la mise à la reproduction de la chèvre et en l'absence de diagnostic échographique, la chèvre est considérée à tort comme gestante.

Les commémoratifs peuvent orienter le diagnostic vers une suspicion de pseudogestation (désaisonnement, mauvais résultats de fécondité, baisse de lait). Par contre la phase de vidange spontanée de l'utérus après plusieurs mois d'évolution est cliniquement visible. Brutalement, un écoulement plus ou moins important se produit à la vulve, parfois sanguinolent. La queue de la chèvre est mouillée. Aucun diagnostic différentiel n'est alors réalisé entre un avortement précoce d'une chèvre gestante et la vidange spontanée d'une chèvre pseudogestante dans la mesure où aucun fœtus, avorton ni placenta n'est retrouvé par l'éleveur.

\section{Diagnostic échographique}

C'est le seul diagnostic de certitude hormis l'autopsie. On observe une image anécho- gène dans un utérus distendu à paroi fine qui semble cloisonné par des parois échogènes minces sans observer ni cotylédon ni placenta ni fœtus. Ces cloisons mobiles correspondent, en fait, aux parois des cornes utérines dilatées et repliées sur elles-mêmes.

La distinction d'avec une gestation est facile après une durée présumée de gestation supérieure à $40 \mathrm{j}$; le foetus est alors bien visible. En revanche, avant $35 \mathrm{j}$, la distinction est délicate. Il est donc conseillé de renouveler l'examen au bout d'un mois.

Lors d'infection du contenu de l'utérus aboutissant à une métrite ou un pyomètre, le contenu de l'utérus apparaît légèrement échogène. Le diagnostic différentiel est parfois délicat.

\section{Autopsies et analyses}

Dix autopsies ont été réalisées après diagnostic échographique de pseudogestation dans les laboratoires vétérinaires départementaux de Bourges et Châteauroux. Des photographies de l'appareil génital complet des chèvres pseudogestantes ont été réalisées. Les analyses bactériologiques, sérologiques et histologiques ont été effectuées par ces laboratoires. Le dosage de la progestérone et de la PSPB sanguine ont été réalisés (Humblot et al, 1990).

Hormis 2 chèvres dont l'utérus s'est vidé entre l'échographie et l'autopsie, les 8 autres appareils génitaux sont remplis d'un liquide stérile clair dont le volume varie de 1-7,2 I. À l'ouverture de l'utérus, on constate que la paroi est mince et qu'aucun placenta ni embryon ne sont présents.

Sur les ovaires dans tous les cas au moins 1 corps jaune est présent ainsi que de nombreux follicules. La progestéronémie était toujours élevée $(>2 \mathrm{ng} / \mathrm{ml}$ ) et la PSPB n'est pas mise en évidence. 


\section{Traitement}

Le traitement le plus utilisé consiste en l'administration de PGF2 $\alpha$ naturelle ou artificielle (cloprostenol, etiproston, etc.) (Smith, 1980; Pieterse et Taverne, 1986; Taverne et al, 1988). On observe l'expulsion du liquide utérin dans les $24-72$ h suivantes. Les chaleurs réapparaissent entre 1-4 j après traitement.

Un essai de traitement a été réalisé sur 12 chèvres pseudogestantes (suite à une mise-bas de novembre) à l'aide d'une injection sous cutanée de $2 \mathrm{mg}$ d'etiproston réalisé début mai :

- 1 chèvre était toujours pseudogestante 1 mois après traitement mais a été fécondée en début de saison sexuelle 4 mois après traitement;

- 11 chèvres se sont vidées dans les jours suivants,

-7 de ces 11 chèvres ont été fécondées entre 42-51 j après traitement et ont mis-bas à contre saison. Parmi ces 7 chèvres, 4 étaient à nouveau pseudogestantes le printemps suivant,

- 2 n'ont été fécondées que 4 mois après traitement (en début de saison sexuelle),

-2 n'ont pas été fécondées et ont été réformées.

\section{ENQUETTE LORS DU DIAGNOSTIC DE GESTATION PAR ÉCHOGRAPHIE}

\section{Matériel et Méthodes}

Les diagnostics échographiques ont été réalisés en 1989 et 1990 dans les élevages caprins du Cher et de l'Indre, sur demande des éleveurs, dans le cadre d'un diagnostic de gestation. Les échographies ont lieu entre 40 et $90 \mathrm{j}$ après la date de saillie ou d'insémination. Elles sont effectuées en moyenne 60 jours après mise à la reproduction, sur des animaux présumés gestants.

En 1989, 5267 échographies ont été réalisées dans 68 élevages; en 1990, 5744 échographies ont été réalisées dans 71 élevages.

L'échographe utilisé est le Shimasonic SDL $32 \mathrm{ER}$, avec une sonde linéaire externe de $85 \mathrm{~mm}$ et 3,5 $\mathrm{MHz}$. Les échographies sont réalisées en série, au sein même de l'élevage, sur des chèvres debout. La sonde, protégée par un film d'huile ou de gel, est apposée dans le creux de l'aine droite, sans rasage préalable.

\section{Résultats}

Incidence de la pseudogestation après mise à la reproduction dans les élevages (tableau I)

Cette pathologie se rencontre dans plus d'un élevage sur deux : $50 \%$ en 1989 et $56 \%$ en 1990 .

Dans plus d'un élevage sur 10 la fréquence de chèvres pseudogestantes est > $5 \%$ :

Tableau I. Incidence de pseudogestations par élevage et par an.

\begin{tabular}{lrr}
$\begin{array}{l}\text { Pourcentage } \\
\text { pseudogestations }\end{array}$ & Nombres d'élevages \\
par élevage & 1989 & 1990 \\
\hline
\end{tabular}

\begin{tabular}{ccc}
\hline 0 & $34(50 \%)$ & $31(43,7 \%)$ \\
de 0 à 3\% & $15(22,1 \%)$ & $18(25,4 \%)$ \\
de 3 à $5 \%$ & $12(17,6 \%)$ & $14(9,7 \%)$ \\
de 5 à $10 \%$ & $5(7,3 \%)$ & $7(9,8 \%)$ \\
$>10 \%$ & $2(3 \%)$ & $1(1,4 \%)$ \\
Totaux & $68(100 \%)$ & $71(100 \%)$ \\
\hline
\end{tabular}


- en 1989, dans 8 élevages sur 68 (soit $11,2 \%$ des élevages enquêtés);

- en 1990, dans 7 élevages sur 71 (soit $10,3 \%$ des élevages enquêtés).

L'incidence de l'affection dans de la population étudiée est respectivement de $2,1 \%$ et $2,9 \%$ des animaux échographiés en 1989 et 1990.

\section{L'âge des animaux (tableau II)}

La pseudogestation se rencontre sur les chèvres ayant déjà mené à terme une gestation mais aussi, et de façon moins fréquente $(p<0,05)$, sur des chevrettes après leur première mise à la reproduction.

Cependant, il existe une différence très fortement significative en $1989(p<0,001)$ et significative en $1990(p<0,05)$ entre le pourcentage de chèvres et de chevrettes mises à la reproduction en monte libre. En 1989 et 1990 respectivement 88 et $78 \%$ des chevrettes de notre échantillon ont été mises à la reproduction en monte libre contre seulement 69 et $71 \%$ des chèvres adultes.

L'incidence de la pseudogestation étant moindre sur les femelles soumises à la monte libre, la conclusion quant à l'influence stricte de l'âge des animaux sur l'affection devient donc plus difficile à analyser dans l'état actuel des données.

\section{Influence de la race}

En 1989, l'incidence dans l'ensemble de l'effectif échographié est très significativement plus élevé dans la race Saanen $(3,6 \%)$ que dans la race Alpine $(1,4 \%)$. En 1990 , on retrouve une situation inverse, sans que la différence soit significative, avec une incidence de $2,4 \%$ dans la race Saanen et $3,4 \%$ dans la race Alpine (tableau III).

En 1989, les élevages présentant un taux de pseudogestation supérieur à $5 \%$ des femelles échographiées se répartissaient de la façon suivante :

-4 élevages de Saanen sur 17 (24\%);

-1 élevage alpin sur $39(3 \%)$;

-2 élevages mixtes sur $12(17 \%)$.

En 1990, les élevages concernés se répartissent de la façon suivante :

- 1 élevage Saanen sur $16(6 \%)$;

-4 élevages alpin sur $37(11 \%)$;

- 3 élevages mixtes sur 18 (17\%).

$\mathrm{Au}$ sein des races, la différence d'incidence est actuellement délicate à interpréter, puisque le rapport entre la race alpine et la race Saanen s'est inversé entre 1989 et 1990. Les élevages Saanen, au contraire de nombreux élevages de chèvres alpines, sont généralement tous des élevages intensifs où sont pratiqués, sur une partie des animaux au moins, le

Tableau II. Incidence de pseudogestations selon l'âge des animaux et les années.

1989

Nombre Nombre de

d'échographies pseudogestantes
1990

\begin{tabular}{ccc}
\hline $\begin{array}{c}\text { Nombre } \\
\text { d'échographies }\end{array}$ & $\begin{array}{c}\text { Nombre de } \\
\text { pseudogestantes }\end{array}$ & (\%) \\
& & \\
4542 & 143 & $3,1 \%$ \\
725 & 9 & $1,2 \%$ \\
5267 & 152 & $2,9 \%$
\end{tabular}

$\begin{array}{lrrllrr}\text { Chèvres } & 4751 & 112 & 2,4 \% & 4542 & 143 & 3,1 \% \\ \text { Chevrettes } & 993 & 7 & 0,7 \% & 725 & 9 & 1,2 \% \\ \text { Total } & 5744 & 119 & 2,1 \% & 5267 & 152 & 2,9 \%\end{array}$


Tableau III. Incidence de pseudogestations selon la race et la période de mise à la reproduction.

\begin{tabular}{|c|c|c|c|c|c|c|c|c|c|}
\hline & \multicolumn{3}{|c|}{ Alpin } & \multicolumn{3}{|c|}{ Saanen } & \multicolumn{3}{|c|}{ Croisés } \\
\hline & Echog & seudog & $\%$ & Echog & Pseudog & $\%$ & Echog & Pseudog & $\%$ \\
\hline \multicolumn{10}{|l|}{1989} \\
\hline mai juin juillet & 1400 & 28 & 2,0 & 694 & 30 & 4,3 & 2276 & 63 & 2,8 \\
\hline aout septembre & 1394 & 16 & 1,2 & 372 & 8 & 2,1 & 2288 & 32 & 1,4 \\
\hline octobre nov déc & 684 & 5 & $0,7^{\prime}$ & 229 & 8 & 3,5 & 1180 & 24 & 2,0 \\
\hline Totaux & 3478 & 49 & 1,4 & 1295 & 46 & 3,6 & 5744 & 119 & 2,1 \\
\hline \multicolumn{10}{|l|}{1990} \\
\hline mai juin juillet & 1425 & 49 & 3,4 & 520 & 18 & 3,5 & 2797 & 89 & 3,2 \\
\hline aout septembre & 852 & 28 & 3,3 & 559 & 8 & 1,4 & 1929 & 41 & 2,1 \\
\hline octobre nov déc & 180 & 6 & 3,3 & 32 & 1 & 3,1 & 267 & 22 & 4,1 \\
\hline Totaux & 2457 & 83 & 3,4 & 1111 & 27 & 2,4 & 5267 & 152 & 2,9 \\
\hline
\end{tabular}

désaisonnement, l'insémination artificielle et la monte en main. Lorsque l'on connaît l'influence que semblent avoir ces deux critères sur la pseudogestation, on comprend pourquoi, sur le plan strictement racial, il est difficile de conclure.

\section{Influence de la période de mise à la reproduction : (tableau III)}

L'incidence des cas de pseudogestation pour l'ensemble de la population étudiée varie de façon hautement significative en $1989(p<0,01)$ et significative en 1990 suivant la période de mise à la reproduction. Les pourcentages maximaux sont observés sur les animaux mis à la reproduction de façon désaisonnée en mai, juin, juillet. Néanmoins en 1990 cette différence n'est pas significative sur la seule population alpine.

\section{Influence du mode de reproduction (tableau IV)}

L'incidence de la pseudogestation pour l'ensemble des animaux varie de façon hautement significative en 1989 ( $p<$ $0,005)$ et significative en $1990(p<0,002)$ suivant le mode de reproduction. Les animaux soumis à une insémination artificielle ou une monte en main précédées d'une synchronisation par pose d'éponge présentent un pourcentage plus élevé de pseudogestation.

\section{CONCLUSION}

La pseudogestation chez la chèvre est une affection qui touche plus d'un élevage caprin sur 2. Dans 1 élevage sur 10 l'incidence de l'affection est $>5 \%$ des chèvres mises à la reproduction et échographiées. Elle correspond à un corps jaune persistant sur l'ovaire qui ne régresse pas malgré l'absence de gestation.

L'augmentation du nombre d'élevages pratiquant le désaisonnement pour avoir du lait d'hiver ainsi que la généralisation du diagnostic échographique dans l'espèce caprine, conduisent à un nombre croissant de diagnostics de cette affection. 
Tableau IV. Incidence de pseudogestations selon la race et le mode de reproduction.

\begin{tabular}{|c|c|c|c|c|c|c|c|c|c|}
\hline \multirow{2}{*}{$\begin{array}{l}\text { Mode de } \\
\text { Reproduction }\end{array}$} & \multicolumn{3}{|c|}{ Alpin } & \multicolumn{3}{|c|}{ Saanen } & \multicolumn{3}{|c|}{ Croisés } \\
\hline & Echog & Pseudog & $\%$ & Echog & Pseudog & $\%$ & Echog & Pseudog & $\%$ \\
\hline \multicolumn{10}{|l|}{1989} \\
\hline Monte libre & 2705 & 41 & 1,5 & 899 & 22 & 2,4 & 4145 & 72 & 1,7 \\
\hline Eponge+lutte main & 311 & 5 & 1,6 & 135 & 16 & 11,9 & 705 & 28 & 4,1 \\
\hline Eponge + IA & 462 & 3 & 0,6 & 261 & 8 & 3,1 & 894 & 19 & 2,1 \\
\hline \multicolumn{10}{|l|}{1990} \\
\hline Monte libre & 1852 & 54 & 2,9 & 712 & 13 & 1,8 & 3774 & 95 & 2,5 \\
\hline Eponge+lutte main & 175 & 9 & 5,1 & 170 & 4 & 2,3 & 725 & 27 & 3,7 \\
\hline Eponge $+1 \mathrm{~A}$ & 430 & 20 & 4,7 & 229 & 10 & 4,4 & 768 & 30 & 3,9 \\
\hline
\end{tabular}

Économiquement, le diagnostic de cette affection peut être réalisé avant la pose d'éponge en vue des inséminations, pour éviter des gaspillages de semence et avant le tarissement, pour laisser les chèvres atteintes en production.

L'administration de prostaglandines constitue un traitement efficace de cette affection.

\section{RÉFÉRENCES}

Cartier S (1986) Synchronisation de l'œstrus chez la chèvre. Diagnostic de gestation. Bull GTV 3, 83-86

Chemineau $P$ (1989) Le désaisonnement des chèvres par la lumière et la mélatonine. Chèvre 171, 18-22

de Montigny $G$ (1985) Les techniques de diagnostic de gestation chez la chèvre. Chèvre $148,33-36$

Etienne P (1991) La pseudogestation chez la chèvre. Rapp stage ENVA, Maisons-Alfort, $58 \mathrm{p}$

Faudell CL (1979) Records of nutritional factors in infertility of goat. Proc 2nd Nat Goat Breeders Conf, Perth, 51-54
Freeman WA (1989) Infertility in goats. Dairy Goat $J 67,784-787$

Humblot $P$, de Montigny $G$, Jeanguyot TN, Tetedoie $F$, Gayen $B$, Thibier $M$, Sasser RG (1990) Pregnancy specific protein $B$ and progesterone concentrations in French Alpine goats throughout gestation. $J$ Reprod Fertil 89, 205-212

Jardon C, Pelzer P (1988) Les scanners arrivent ! Pâtre 338,17-21

Lyngset $O$ (1968) Studies on reproduction in the goat. V. Pathological conditions and malformations of the genital organs of the goat. Acta Vet Scand 9, 364-375

Malher X, Younes AB (1987) Les facteurs zootechniques de l'infécondité dans l'espèce caprine. Rec Méd Vét 163, 831-838

Mews AR (1981) Breeding and infertility in goats. Br Goat Soc Year Book

Mialot JP, Levy I, Emery P (1991a) Echographie et gestion des troupeaux caprins. Rec Med Vet 168, 399-406

Mialot JP, Saboureau $L$, Guéraud JM, Prengere E, Parizot D, Pirot G, Duquesnel R, Petat M, Chemineau P (1991b) La pseudogestation chez la chèvre. Observations préliminaires. Rec Med Vet 167, 383-390

Pieterse MC, Taverne MAM (1986) Hydrometra in goats: diagnosis with real-time ultrasound and treatment with prostaglandin or oxytocin. Theriogenology 26, 813-821 
Saboureau L (1990) Stage au GDS. du Cher. Rapp de stage ENVA, Maisons-Alfort, $70 p$

Smith MC (1980) Caprine reproduction in current therapy in theriogenology (Morrow, ed) WB Saunders, Philadelphia, PA 1143

Tainturier D, Lijour L, Chaari M, Sardjana KW, Lenet JL (1983) Diagnostic de la gestation chez la chèvre par échotomographie. Rec Méd Vét 134, 597-599

Taverne MAM, Lavoir MC, Bevers MM, Pieterse MC, Dieleman SJ (1988) Peripheral plasma prolactin and progesterone levels in pseudopregnant goats during bromocryptine treatment. Theriogenology $30,777-783$ 